\title{
CARACTERÍSTICAS SEMINAIS DE GALOS ALIMENTADOS COM RAÇÕES SUPLEMENTADAS COM DIFERENTES ÓLEOS E NÍVEIS DE VITAMINA E
}

\author{
Seminal characteristics roosters fed diets supplemented with different oils and vitamin $E$ \\ Carolina Elizabeth Orihuela Rodenas ${ }^{1}$, Luis David Solis Murgas², Mônica Patrícia Maciel ${ }^{3}$, \\ Julliana Melo Ferraz ${ }^{4}$, Maria Carolina Ribeiro ${ }^{5}$, Antonio Gilberto Bertechini ${ }^{6}$, \\ Rilke Tadeu Fonseca de Freitas ${ }^{7}$, Elias Tadeu Fialho ${ }^{8}$
}

\section{RESUMO}

Realizou-se este trabalho no setor de avicultura de DZO-UFLA, durante o período de junho a outubro de 2003. Foram utilizados 112 galos reprodutores da linhagem Lohmann- LSL, de 16 até 32 semanas de idade. As aves foram distribuídas num delineamento em blocos ao acaso, em arranjo fatorial 3x2 + 1, com três tipos de óleo (Soja, Canola e Girassol), e dois níveis de vitamina E (200 e $400 \mathrm{mg} / \mathrm{kg}$ de ração) e o controle (sem óleo e $0 \mathrm{mg}$ de suplementação de vitamina E). As rações experimentais foram elaboradas à base de milho e farelo de soja. Nas $28^{\mathrm{a}}$ e $29^{\mathrm{a}}$ semanas foram realizadas coletas de sêmen para avaliação da motilidade, volume do sêmen, concentração, número de células totais e a morfologia espermática. As análises foram realizadas com utilização do programa SAEG. Foi observada interação significativa $(\mathrm{P}=0,08)$ entre as fontes de óleos testadas e os níveis de vitamina $\mathrm{E}$, para a motilidade espermática na $28^{\mathrm{a}}$ semana de idade. Não foi observada diferença significativa $(\mathrm{P}>0,05)$ entre as fontes de óleo nem entre os níveis de vitamina $\mathrm{E}$ para nenhuma das variáveis estudadas. Conclui-se que qualquer fonte de óleo das utilizadas neste estudo pode ser adicionada à ração de galos reprodutores sem necessidade de elevados níveis de vitamina $\mathrm{E}$.

Termos para Indexação: Galos reprodutores, ácidos graxos essenciais, vitamina E.

\begin{abstract}
The objective of this study was evaluate breeding and productive performance of Lohmann- LSL roosters. A total of 112 breeding roosters from 16 to 32 weeks of age were allotted to a randomized block design in a $3 \times 2+1$ factorial arrangement, with three sources of oil (soybean, canola and sunflower in 3\%of inclusion ) and two levels of antioxidant (200 and $400 \mathrm{mg}$ of vitamin E/kg of diet) and the control diet (without oil and $0 \mathrm{mg}$ of supplementation of vitamin $\mathrm{E}$ ). The experimental diets were made based on corn and soybean meal. At 28 and 29 weeks, semen collection was performed for evaluation of sperm motility, semen volume, concentration and number of total cells. At 29 weeks of age, semen collection was performed to evaluate sperm morphology, rate of osmotic resistance and heat-resistance of spermatozoa. Four roosters per treatment were slaughtered at 30 weeks of age for removal of the testes which were weighted and a sample was obtained for evaluation of the thickness of the seminiferous epithelium, diameter and the population of seminiferous tubule cells. Blood samplings were collected for evaluation of triglycerides and cholesterol. During the experimental period from 16 to 30 weeks, feed intake and total weight gain were evaluated. The true fertility test was performed at 32 weeks of age. The data were evaluated by analysis of variance using the SAEG program. Significant interaction $(\mathrm{P}<0.05)$ was found between sources of oil and the levels of vitamin $\mathrm{E}$ as well as among the sources of oils for total weight gain, seminiferous epithelium thickness and body weight at 30 weeks of age. Significant difference $(\mathrm{P}<0.05)$ was verified among the levels of vitamin $\mathrm{E}$ for the levels of triglycerides, cholesterol and body weight at 30 weeks of age. It should be concluded that any ource of oil used in this study may be added to the breeding rooster diet without the need to increase the levels of vitamin $\mathrm{E}$.
\end{abstract}

Index terms: oils, reproductive performance, vitamin, fatty acids.

(Recebido para publicação em 27 de abril de 2004 e aprovado em 30 de novembro de 2004)

\section{INTRODUÇÃO}

Dentre os fatores que afetam a fertilidade dos machos destacam-se a temperatura ambiente, o fotoperíodo, patologias, manejo e nutrição. A produção de espermatozóides e a fertilidade são influenciadas pela alimentação, tanto no período de crescimento, quanto no de produção (ETCHES, 1996).

Entre os vários nutrientes que exercem efeito sobre a biologia dos espermatozóides estão os lipídeos (KELSO, 1997). Entretanto, há poucas pesquisas relacionadas com a sua influência sobre a fertilidade em aves.

\footnotetext{
1. Zootecnista, Mestranda - Departamento de Zootecnia/Universidade Federal de Lavras/UFLA - Caixa Postal 3037 - 37.200-000 - Lavras, MG rodenasorihuela@hotmail.com

2. Médico veterinário, D. Sc. Fisiologia Animal, Professor Adjunto Departamento de Medicina Veterinária/UFLA - Ismurgas@ufla.br

3. Zootecnista, Doutoranda - Departamento de Zootecnia//UFLA - mpmaciel@hotmail.com

4. Acadêmica do curso de medicina veterinária/UFLA - juferraz@hotmail.com.br

5. Acadêmica do curso de medicina veterinária/UFLA - mcribeiro@hotmail.com.br

6. Zootecnista, D.Sc. Professor Adjunto - Departamento de Zootecnia/UFLA - beterchini@ufla.com.br

7. Zootecnista, D.Sc. Professor Adjunto - Departamento de Zootecnia/UFLA - rilke@ufla.com.br

8. Engenheiro agrônomo, Professor Titular - Departamento de Zootecnia/UFLA - fialho@ufla.com.br
} 
Além da função energética, os lipídeos também são componentes celulares de membranas biológicas. Em todas as espécies (MARTIN RILLO et al., 1996), os fosfolipídios são os principais componentes lipídicos dos espermatozóides, caracterizados por conterem grandes quantidades de ácidos graxos poliinsaturados - PUFAs, o que sugere que a composição de lipídios e ácidos graxos dos espermatozóides pode ser um fator determinante das taxas de fertilidade. Segundo Kelso (1997), os espermatozóides de galos contêm principalmente ácidos graxos insaturados de cadeias longas 20 e 22 carbonos da série ômega 6. Os PUFAs da série ômega 3 e ômega 6 são ácidos graxos essenciais, uma vez que não podem ser sintetizados pelos animais e precisam ser fornecidos pela ração. Os PUFAs são materiais oxidáveis, por possuírem duplas ligações que formam radicais livres ao se juntarem ao $\mathrm{O}_{2}$ metabólico, dando origem aos peróxidos. Um dos antioxidantes mais utilizados na membrana é a vitamina E, que neutraliza os radicais livres, assim evita-se dano celular, o qual ocasiona desarranjos metabólicos como por exemplo, a inibição de atividades enzimáticas (MCDOWELL, 1989).

Objetivou-se com este trabalho avaliar as características seminais de galos Lohmann-LSL, alimentados com rações contendo óleos de diferentes fontes e níveis de vitamina $\mathrm{E}$.

\section{MATERIAL E MÉTODOS}

O experimento foi conduzido no Setor de Avicultura do Departamento de Zootecnia da Universidade Federal de Lavras, durante o período de 17 semanas, de junho a outubro de 2003, com temperaturas médias máxima e mínimas de 24,9 e $12,0{ }^{\circ} \mathrm{C}$, respectivamente. As análises laboratoriais foram realizadas no Laboratório de Fisiologia e Farmacologia do Departamento de Medicina Veterinária.

Foram utilizados 112 galos, da linhagem Lohman LSL, procedentes da Granja Planalto-Uberlândia MG, com 16 semanas de idade e peso inicial médio de 1830/g $( \pm 98,8)$, sendo alojados em gaiolas metálicas individuais. As rações experimentais foram isoprotéicas (17\%) e isoenergéticas (2965kcal de EM/Kg), formuladas à base de milho e farelo de soja e suplementadas com minerais e vitaminas. Os níveis nutricionais foram os recomendados para a linhagem e a composição dos ingredientes segundo Rostagno et al. (1992). $\mathrm{O}$ ajuste da vitamina $\mathrm{E}$ foi feito alterando-se a proporção de caulim e vitamina $\mathrm{E}$ nas rações. $\mathrm{O}$ consumo de ração foi à vontade.
Todos os galos foram treinados para coleta de sêmen a partir da $16^{\mathrm{a}}$ semana de idade. A coleta foi realizada pelo método de massagem abdominal proposto por Burrows e Quinn (1935). Duas semanas antes da coleta, os animais foram submetidos a uma "toillete", retirando-se as penas da região pericloacal com uso de tesoura, para evitar a contaminação do sêmen coletado. Durante a fase experimental, foram realizadas duas coletas seminais na $28^{\mathrm{a}}$ e $29^{\mathrm{a}}$ semana de idade dos animais, por serem idades do começo do ciclo reprodutivo.

Os galos foram distribuídos num delineamento experimental em blocos ao acaso, sendo 28 aves por bloco (peso), com a seguinte distribuição: peso 1(1590$1715 \mathrm{~g})$, peso 2 (1720-1845g), peso 3 (1850-1975g) e peso 4 (1980-2105g), com arranjo fatorial de $3 \times 2+1$, com três tipos de óleo aos 3\% (soja, canola, girassol), dois níveis de vitamina E (200 e $400 \mathrm{mg} / \mathrm{kg}$ de alimento) e o tratamento-controle sem óleo nem suplementação de vitamina E. Utilizaram 16 repetições por tratamento, sendo cada galo uma unidade experimental.

Os parâmetros seminais avaliados foram motilidade espermática, volume do ejaculado, concentração espermática, número total de células e anormalidades espermáticas. A motilidade geral foi avaliada segundo escala de 0 a 100 . Retirou-se uma gota de sêmen imediatamente após a coleta, colocando-a sobre lâmina recoberta por lamínula previamente aquecida à temperatura de $40^{\circ} \mathrm{C}$ para a leitura no microscópio óptico (40X). $\mathrm{O}$ volume foi medido diretamente da seringa de coleta com graduação de $0,1 \mathrm{ml}$.

Para análise de concentração espermática, foi retirada uma amostra de $10 \mu \mathrm{l}$ de sêmen para ser adicionado a $4 \mathrm{~mL}$ de solução de formol citrato, sendo a contagem realizada com o uso de hemocitômetro (câmara de Newbauer) na diagonal com o resultado expresso em número de células por $\mathrm{ml}$ de sêmen. $\mathrm{O}$ número total de células foi calculado pela multiplicação da concentração e volume do ejaculado, segundo a metodologia de Martin Rillo et al. (1996).

Para avaliação das anormalidades dos espermatozóides, $1 \mu \mathrm{l}$ de sêmen foi adicionado a $250 \mu \mathrm{l}$ de solução de formol citrato. Seguidamente, no microscópio de contraste de fase com aumento de 1000X, realizou-se a contagem de 100 células, expressando as alterações morfológicas em percentagem. As alterações espermáticas avaliadas foram alterações de cabeça, cauda, presença de gota e alterações totais.

As análises estatísticas foram realizadas com o programa SAEG (Sistema de Análises Estatísticas e Genéticas) desenvolvido pela UFV. 
Para verificar a normalidade das variáveis, utilizou-se o teste de Lillefors. Houve transformação de dados para ajustar a normalidade para as seguintes variáveis: volume seminal para raiz de $\mathrm{X}$; número total de espermatozóides para raiz de $\mathrm{X}$, morfologia espermática para Arcoseno raiz de $\mathrm{X} / 10$.

Para as variáveis avaliadas, utilizou-se o seguinte modelo estatístico:

em que:

$$
\mathrm{Y}_{\mathrm{ijk}}=\mu+\mathrm{Oi}+\mathrm{V}_{\mathrm{j}}+(\mathrm{OV})_{\mathrm{ij}}+\mathrm{B}_{\mathrm{k}}+\mathrm{e}_{\mathrm{ijk}}
$$

$\mathrm{Y}_{\mathrm{ijk}}=$ característica avaliada referente à fonte de óleo $\mathrm{i}$, no nível $\mathrm{j}$ de vitamina $\mathrm{E}$ do bloco $\mathrm{k}$;

$\mu=$ média geral;

$\mathrm{O}_{\mathrm{i}}=$ efeito do tipo de óleo $\mathrm{i}$, sendo $\mathrm{i}=1,2$ e 3 ;
$\mathrm{V}_{\mathrm{j}}=$ efeito do nível $\mathrm{j}$ de vitamina $\mathrm{E}$, sendo $\mathrm{j}=1$ e 2;

$(\mathrm{OV})_{\mathrm{ij}}=$ efeito da interação da fonte de óleo i e o nível $\mathrm{j}$ de vitamina $\mathrm{E}$;

$\mathrm{B}_{\mathrm{k}}=$ efeito do bloco $\mathrm{k}$, sendo $\mathrm{k}=1,2,3$ e 4 ;

$\mathrm{e}_{\mathrm{ijk}}=$ erro experimental associado a cada observação.

Para comparar as médias, utilizou-se o teste de agrupamento Scott Knott.

\section{RESULTADOS E DISCUSSÃO}

\section{Volume seminal e motilidade espermática}

Os resultados de volume do sêmen produzido e da motilidade espermática, nas $28^{\mathrm{a}}$ e $29^{\mathrm{a}}$ semana de idade dos reprodutores, estão apresentados nas tabelas $1 \mathrm{e}$ 2 , respectivamente.

TABELA 1 - Volume seminal de galos Lohman-LSL, em ml, na $28^{\mathrm{a}}$ e $29^{\mathrm{a}}$ semanas de idade, alimentados com diferentes óleos e níveis de vitamina E.

\begin{tabular}{|c|c|c|c|}
\hline \multirow{2}{*}{ Tipo de Óleo } & \multicolumn{3}{|c|}{ Nível de Vitamina E (mg/kg) } \\
\hline & 200 & 400 & Média \\
\hline \multicolumn{4}{|c|}{ VOLUME DE SÊMEN NA $28^{\mathrm{a}}$ SEMANA DE IDADE (mL) } \\
\hline Soja & 0,13 & 0,13 & 0,13 \\
\hline Canola & 0,11 & 0,10 & 0,10 \\
\hline Girassol & 0,13 & 0,13 & 0,13 \\
\hline Média & 0,12 & 0,12 & 0,11 \\
\hline Controle & & & 0,10 \\
\hline $\mathrm{CV}(\%)$ & & 27,9 & \\
\hline \multicolumn{4}{|c|}{ VOLUME DE SÊMEN NA 29a SEMANA DE IDADE (mL) } \\
\hline Soja & 0,15 & 0,17 & 0,16 \\
\hline Canola & 0,12 & 0,13 & 0,13 \\
\hline Girassol & 0,17 & 0,19 & 0,18 \\
\hline Média & 0,15 & 0,16 & 0,15 \\
\hline Controle & & & 0,14 \\
\hline $\mathrm{CV}(\%)$ & & 28,2 & \\
\hline
\end{tabular}

Ciênc. agrotec., Lavras, v. 29, n. 1, p. 160-167, jan./fev. 2005 
TABELA 2 - Motilidade espermática de galos Lohman-LSL, em porcentagem, na $28^{\mathrm{a}}$ e $29^{\mathrm{a}}$ semana de idade, alimentados com diferentes óleos e níveis de vitamina $\mathrm{E}$.

\begin{tabular}{|c|c|c|c|}
\hline \multirow{2}{*}{ Tipo de Óleo } & \multicolumn{3}{|c|}{ Nível de Vitamina E (mg/kg) } \\
\hline & 200 & 400 & Média \\
\hline \multicolumn{4}{|c|}{ MOTILIDADE NA 28ª SEMANA DE IDADE (\%) } \\
\hline Soja & 84,5 & $83,3 b$ & 83,9 \\
\hline Canola & 86,8 & $83,3 b$ & 85,0 \\
\hline Girassol & 81,6 & $87,3 \mathrm{a}$ & 84,3 \\
\hline Média & 84,3 & 84,6 & 83,8 \\
\hline Controle & & & 80,0 \\
\hline CV (\%) & & 12,0 & \\
\hline \multicolumn{4}{|c|}{ MOTILIDADE ÀS $29^{\mathrm{a}}$ SEMANA DE IDADE (\%) } \\
\hline Soja & 86,8 & 80,4 & 83,5 \\
\hline Canola & 85,4 & 82,1 & 83,8 \\
\hline Girassol & 85,8 & 88,3 & 87,1 \\
\hline Média & 85,6 & 83,6 & 84,3 \\
\hline Controle & & & 81,6 \\
\hline $\mathrm{CV}(\%)$ & & 10,2 & \\
\hline
\end{tabular}

Médias seguidas de letras distintas na mesma coluna diferem pelo teste Scott-Knott $(P=0,08)$

Não foi observada interação significativa $(\mathrm{P}>0,05)$ entre os tipos de óleo e os níveis de vitamina para o volume de sêmen nas duas idades. As fontes de óleo e os níveis de vitamina $\mathrm{E}$ na ração não influenciaram $(\mathrm{P}>0,05) \mathrm{o}$ volume de sêmen nas idades de 28 e 29 semanas.

$\mathrm{O}$ volume de sêmen observado nos animais, neste trabalho, está de acordo com Rouvier et al. (1984), citados por Etches (1996), os quais relataram uma variação entre 0,05 e $0,30 \mathrm{ml}$ para galos de linhagens leves. Em mamíferos, a maior proporção do volume seminal é originária das glândulas acessórias (HAFEZ, 1982). Por outro lado, em aves, a ausência de vesículas seminais e glândula prostática resulta em reduzido volume seminal, o qual é composto basicamente de frutose, fosfatidilcolina e alguns eletrólitos (PARKHURST e MOUNTNEY, 1988).

Para a motilidade espermática nota-se uma interação significativa $(\mathrm{P}<0,05)$ entre os tipos de óleo e os níveis de vitamina $\mathrm{E}$ na $28^{\mathrm{a}}$ semana de idade, observando-se que, no nível de $400 \mathrm{mg}$ de vitamina $\mathrm{E}$, a ração suplementada com óleo de girassol promoveu uma motilidade espermática maior $(\mathrm{P}<0,10)$.

A motilidade espermática na idade de 29 semanas não apresentou interação significativa $(\mathrm{P}>0,05)$ das fontes de óleo e níveis de vitamina E. Entretanto, o óleo de girassol apresentou um valor numérico maior, não-significativo $(\mathrm{P}>0,10)$ para esse parâmetro no nível de $400 \mathrm{mg}$ de vitamina E, acompanhando o resultado na $28^{\mathrm{a}}$ semana de idade.

Segundo Celeghini et al. (2000), a motilidade espermática e o volume seminal não se correlacionam com o peso corporal em galos da linhagem AgRoss. Diferente desse resultado, observou-se neste estudo que o peso influenciou significativamente $(\mathrm{P}<0,01)$ o volume seminal na $29^{a}$ semana de idade, com $2145( \pm 115 \mathrm{~g})$ de peso corporal, comparado com a $28^{\mathrm{a}}$ semana de idade, 
com um peso corporal de $2098( \pm 117 \mathrm{~g})$. Esses resultados são semelhantes aos observados por Rouvier et al. (1984), citados por Etches (1996), os quais observaram um aumento do volume seminal com o aumento do peso corporal dependendo da linhagem dos reprodutores.

Segundo Zanini (2001), o óleo de girassol apresenta um teor bastante elevado de ácidos graxos poliinsaturados, o que pode ter promovido uma viabilidade maior das células com conseqüente maior motilidade. Neste experimento, a suplementação de vitamina E na ração no nível de $400 \mathrm{mg} / \mathrm{kg}$ no óleo de girassol pode melhorar a motilidade espermática pela proteção do epitélio germinativo testicular contra a peroxidação dos lipídeos de membrana. Uma vez que o óleo de girassol apresenta uma maior proporção de ácidos graxos poliinsaturados (ZANINI et al., 2003) em relação ao óleo de soja e canola, a exigência por antioxidante se torna maior.

\section{Concentração espermática e número total de células}

Os resultados de concentração espermática e número total de espermatozóides estão apresentados nas Tabelas 3 e 4, respectivamente.
Não foi observada interação significativa ( $\mathrm{P}>0,05)$ entre os tipos de óleo e os níveis de vitamina $E$ utilizados neste experimento sobre essas variáveis, nas idades de 28 e 29 semanas. Entretanto, a concentração espermática observada no nível $200 \mathrm{mg}$ de vitamina $\mathrm{E}$ com óleo de canola apresentou valor numérico maior, não-significativo $(\mathrm{P}>0,05)$, comparado com os óleos de soja e girassol na $29^{\mathrm{a}}$ semana de idade. Biologicamente, esse valor representa um aumento de milhões de espermatozóides nos animais que receberam esse tratamento.

A relação ácidos graxos insaturados/saturados observada no óleo de canola é maior do que para os óleos de girassol e soja (ZANINI et al., 2003). Esse fator pode ter promovido uma melhor relação entre os ácidos graxos das séries ômega 6 e ômega 3.

Diferentemente do volume seminal, a concentração espermática apresenta correlação significativa de 0,29 com o peso corporal em galos (CELEGHINI et al., 2000). Concordando com os resultados obtidos neste trabalho, em que se observou um efeito significativo do peso corporal sobre a concentração espermática. Os animais mais pesados apresentaram maior concentração espermática, independentemente do tratamento utilizado.

TABELA 3 - Concentração espermática de galos Lohman-LSL, nas $28^{\mathrm{a}}$ e $29^{\mathrm{a}}$ semanas de idade, alimentados com diferentes óleos e níveis de vitamina $\mathrm{E}$.

\begin{tabular}{|c|c|c|c|}
\hline \multirow{2}{*}{ Tipo de Óleo } & \multicolumn{3}{|c|}{ Nível de Vitamina E (mg/kg) } \\
\hline & 200 & 400 & Média \\
\hline \multicolumn{4}{|c|}{ CONCENTRAÇÃO ESPERMÁTICA NA $28^{\text {a }}$ SEMANA DE IDADE (x $10^{9}$ esperm/ml) } \\
\hline Soja & 1,42 & 1,46 & 1,44 \\
\hline Canola & 1,62 & 1,20 & 1,41 \\
\hline Girassol & 1,32 & 1,46 & 1,39 \\
\hline Média & 1,45 & 1,37 & 1,39 \\
\hline Controle & & & 1,23 \\
\hline $\mathrm{CV}(\%)$ & & 60,5 & \\
\hline \multicolumn{4}{|c|}{ CONCENTRAÇÃO ESPERMÁTICA NA 29a SEMANA DE IDADE (x $10^{9}$ esperm/ml) } \\
\hline Soja & 1,78 & 2,03 & 1,90 \\
\hline Canola & 2,44 & 1,70 & 2,05 \\
\hline Girassol & 1,83 & 1,68 & 1,75 \\
\hline Média & 2,02 & 1,80 & 1,87 \\
\hline Controle & & & 1,70 \\
\hline CV $(\%)$ & & 60,8 & \\
\hline
\end{tabular}

Ciênc. agrotec., Lavras, v. 29, n. 1, p. 160-167, jan./fev. 2005 
TABELA 4 - Número de células espermáticas totais em galos Lohman- LSL, nas $28^{\mathrm{a}}$ e $29^{\mathrm{a}}$ semanas de idade, alimentados com diferentes óleos e níveis de vitamina E.

\begin{tabular}{|c|c|c|c|}
\hline \multirow{2}{*}{ Tipo de Óle } & \multicolumn{3}{|c|}{ Nível de Vitamina E (mg/kg) } \\
\hline & 200 & 400 & Média \\
\hline \multicolumn{4}{|c|}{ NÚMERO DE CÉLULAS TOTAIS NA $28^{\mathrm{a}}$ SEMANA DE IDADE (x $10^{7}$ esperm) } \\
\hline Soja & 18,8 & 20,4 & 19,6 \\
\hline Canola & 18,8 & 15,6 & 17,2 \\
\hline Girassol & 21,2 & 21,9 & 21,5 \\
\hline Média & 19,6 & 19,3 & 18,7 \\
\hline Controle & & & 14,4 \\
\hline $\mathrm{CV}(\%)$ & & $C V(\%)$ & \\
\hline \multicolumn{4}{|c|}{ NÚMERO DE CÉLULAS TOTAIS NA 29a SEMANA DE IDADE (x $10^{7}$ esperm) } \\
\hline Soja & 23,0 & 35,0 & 29,2 \\
\hline Canola & 35,9 & 24,8 & 30,4 \\
\hline Girassol & 32,8 & 29,1 & 31,1 \\
\hline Média & 30,6 & 29,6 & 29,3 \\
\hline Controle & & & 24,0 \\
\hline CV $(\%)$ & & 43,2 & \\
\hline
\end{tabular}

Surai et al. (2000) mencionam que o resultado da concentração espermática está diretamente relacionado ao volume seminal em galos. Da mesma forma, Celeghini et al. (2000) observaram uma correlação positiva significativa entre o volume seminal, concentração espermática e número de células totais em galos.

Em trabalhos realizados com suínos por Brezezinska-Slebodzinska et al. (1995), observou-se que a concentração espermática aumentou com a suplementação de vitamina $\mathrm{E}$ na ração de suínos. Por outro lado, segundo Klasing (1998), altos níveis de vitamina $\mathrm{E}$ podem causar deficiência de outras vitaminas lipossolúveis, como, por exemplo, a vitamina $\mathrm{A}$, que tem um papel importante na proteção do epitélio germinativo e manutenção da integridade das células produtoras de testosterona nos machos.

\section{Morfologia espermática}

Os dados de morfologia espermática, incluindo anormalidades de cabeça, cauda, presença de gota e alterações totais, estão apresentados na Tabela 5. Não se observou efeito significativo $(\mathrm{P}>0,05)$ do peso corporal dos animais sobre estes parâmetros, e a interação entre as fontes de óleo e os níveis de vitamina E também não foi significativa $(\mathrm{P}>0,05)$ para esses parâmetros.

As anormalidades espermáticas são incompatíveis com a fertilidade, a despeito dos parâmetros físicos do sêmen. Em mamíferos, a porcentagem de alterações morfológicas totais é um parâmetro importante para determinar a escolha dos melhores reprodutores. Murgas (1999) relata que para manter uma taxa de fertilidade constante, a porcentagem total de espermatozóides com alterações morfológicas, em suínos, não deve ultrapassar $20 \%$ do número total de espermatozóides por ejaculado.

Trabalhos abordando a morfologia espermática em galos são bastante escassos e, em menor número ainda são os que relacionam esses achados aos problemas reprodutivos de um plantel.

Em trabalho realizado por Jaenisch (1998) utilizando galos da linhagem de corte Peterson, notou-se que as variações no peso corporal comprometem a morfologia espermática de galos, sendo mais evidente em galos com excesso de peso. Esses autores observaram um máximo de $11,21 \%$ de alterações espermáticas totais nos galos mais pesados. A média de alterações totais observada no presente trabalho foi de $10,1 \%$. 
TABELA 5 - Morfologia espermática de galos Lohman-LSL, em porcentagem na $29^{\text {a }}$ semana de idade, alimentados com diferentes óleos e níveis de vitamina $\mathrm{E}$.

\begin{tabular}{|c|c|c|c|}
\hline \multirow{2}{*}{ Tipos de Óleo } & \multicolumn{3}{|c|}{ Nível de Vitamina E (mg/kg) } \\
\hline & 200 & 400 & Média \\
\hline \multicolumn{4}{|c|}{ CABEÇAS ANORMAIS DOS ESPERMATOZÓIDES (\%) } \\
\hline Soja & 2,4 & 3,8 & 3,1 \\
\hline Canola & 3,1 & 2,1 & 2,6 \\
\hline Girassol & 3,2 & 2,1 & 2,6 \\
\hline Média & 2,9 & 2,7 & 2,9 \\
\hline Controle & & & 3,4 \\
\hline CV $(\%)$ & & 44,8 & \\
\hline \multicolumn{4}{|c|}{ CAUDAS ANORMAIS DOS ESPERMATOZÓIDES (\%) } \\
\hline Soja & 1,8 & 2,3 & 2,1 \\
\hline Canola & 2,2 & 2,2 & 2,2 \\
\hline Girassol & 2,5 & 2,3 & 2,4 \\
\hline Média & 2,2 & 2,3 & 2,3 \\
\hline Controle & & & 3,1 \\
\hline $\mathrm{CV}(\%)$ & & 51,7 & \\
\hline \multicolumn{4}{|c|}{ PRESENÇA DE GOTA NOS ESPERMATOZÓIDES \% } \\
\hline Soja & 4,5 & 6,6 & 5,5 \\
\hline Canola & 5,2 & 4,6 & 4,9 \\
\hline Girassol & 3,5 & 4,5 & 4,0 \\
\hline Média & 4,4 & 5,2 & 4,9 \\
\hline Controle & & & 5,3 \\
\hline CV $(\%)$ & & 29,26 & \\
\hline \multicolumn{4}{|c|}{ TOTAL DE ESPERMATOZÓIDES ANORMAIS \% } \\
\hline Soja & 8,8 & 12,8 & 10,8 \\
\hline Canola & 10,4 & 8,8 & 9,5 \\
\hline Girassol & 9,2 & 8,8 & 9,0 \\
\hline Média & 9,5 & 10,1 & 10,1 \\
\hline Controle & & & 11,8 \\
\hline CV (\%) & & 24,8 & \\
\hline
\end{tabular}

Ciênc. agrotec., Lavras, v. 29, n. 1, p. 160-167, jan./fev. 2005 


\section{CONCLUSÕES}

Nas condições em que foi realizado este trabalho, pode-se concluir que qualquer tipo de óleo dos utilizados neste estudo pode ser adicionado à ração de galos reprodutores, sem afetar as características seminais e sem necessidade de se aumentar os níveis de vitamina $\mathrm{E}$.

\section{REFERÊNCIAS BIBLIOGRÁFICAS}

BREZEZINSKA-SLEBODZINSKA, E. et al. Antioxidant effect of vitamin $\mathrm{E}$ and glutathione on lipid peroxidation in boar semen plasma. Biological Trace Elementary Research, Clifton, v. 47, n. 1-3, p. 69-74, 1995.

BURROWS, W. R.; QUINN, A. J. P. Method of obtaining spermatozoa from the domestic fowl. Poult Science, Champaign, v. 14, p. 253, 1935.

CELEGHINI, E. C. C. et al. Correlações entre as características seminais, parâmetros testiculares (peso) e histologia e peso corporal em galos. Brazilian Journal of Poultry Science, Campinas, v. 1, p. 56, 2000.

ETCHES, R. J. Reproducción Aviar. Zaragoza: Acribia, 1996. $339 \mathrm{p}$.

HAFEZ, E. S. E. Reprodução animal. 4. ed. São Paulo: Manole, 1982.720 p.

JAENISCH, F. R. F. Morfologia espermática em galos com diferentes pesos corporais. In: REUNIÃO ANUAL DA SOCIEDADE BRASILEIRA DE ZOOTECNIA, 35., 1998, Fortaleza, CE. Anais... Fortaleza: SBZ, 1998. p. 401-403.

KELSO, K. A. et al. Effect of dietary supplementation with $\alpha$ Linolenic acid on the phospholipids fatty acid composition and quality of spermatozoa in cockerel from 24 to 72 week of age. Journal of Reproduction and Fertility, Cambridge, v. 110, p. 53-59, 1997.
KLASING, K. C. Comparative avian nutrition. UK: Cab International, 1998. $350 \mathrm{p}$.

MCDOWELL, L. R. Vitamin in animal nutrition: comparative aspect to human nutrition. Washington: Academic, 1989. 486 p.

MARTIN RILLO, S. et al. Bora semen evaluation in practice. Reproduction Domestics Animal,[S.1.], v. 31, n. 4, p. 519-526, 1996.

MURGAS, L. D. S. Desempenho reprodutivo de varrões híbridos alimentados com rações suplementadas com óleo de soja como fonte de ácidos graxos. 1999. 111 p. Tese (Doutorado em Zootecnia) - Universidade Federal de Lavras, Lavras, 1999.

PARKHURST, C. R.; MOUNTNEY, G. J. Poultry meat and egg production. New York: Avi Book, 1988. $294 \mathrm{p}$.

ROSTAGNO, H. S. et al. Composição de alimentos e exigências nutricionais de aves e suínos: tabelas brasileiras. Viçosa: UFV, 1992. 60 p.

SURAI, P. F. et al. Effect of long-term supplementation with arachidonic or docosahexaenoic acids on sperm production in broiler chicken. Journal of Reproduction and Fertility, Cambridge, v. 120, p. 257-264, 2000.

ZANINI, S. F. Fontes de óleo e níveis de suplementação de vitamina $E$ na ração sobre o desempenho produtivo e reprodutivo de galos leves. $2001.115 \mathrm{f}$. Tese (Doutorado em Zootecnia) - Universidade Federal de Viçosa, Viçosa, 2001.

ZANINI, S. F. et al. Fontes de óleo e níveis de vitamina E sobre desempenho produtivo e reprodutivo de galos. Revista Brasileira de Ciência Avícola, Campinas, n. 5, p. 71, 2003. 